\title{
Image Generation Method Based on Image Edge Information
}

\author{
Yanqiu Liu ${ }^{1}$ and Xiuhui Wang ${ }^{2}$ \\ China Jiliang University, Hangzhou 310018, china \\ 149742264@qq.com, 2232926357@qq.com
}

\begin{abstract}
An algorithm was proposed to generate automatically embedded image. With the use of outline matching and shape deformation, the proposed method can insert an interior object with partial deformation into an outer object image, having better results by considering the cavity of the outer object. The embedded object is easily observed because its color is opposite to the outer object while the outer object's cavity can be part of the interior object. By designing method which is applied to express object's edge strength, the matching result of the algorithm can be optimized.
\end{abstract}

Keywords: Edge matching, region classification, image processing, sketch image rendering

\section{Introduction}

Human visual process includes two steps:

(1) Human eyes accept and analyze optical images and then form perception, as to identify object shape and space and further on to obtain its formal and spatial changes;

(2) Human brain analyzes the received object and gets four main information i.e. object color, shape, space and variation. Based on those data, we can recognize different objects and react accordingly to them [1-3].

Around the year of 1910, a few German scientists Wertheimer, Kohler and Koffka proposed Gestalt theory. The theory thinks that as far as human perception is concerned, there's one shape which is visually the simplest and most complete, which is called "whole form". No matter what object is seen, human visual system would automatically try to combine them to get a best whole form. The major contribution of the theory is to discover and comprehend those whole forms. When information is carried from sensory organs to brain, the brain begins immediately analyzing and sorting out. In the process, to give assistance to human's perception, in the brain a total form will naturally show up [45].

In real world, outside sceneries are constantly varying. An object visible at previous moment may have been masked at this moment, so human vision would supplement the missing partition of an object as per experience. Particularly when identifying complicated objects, human beings discern them subject to their parts with the application of priori knowledge. For familiar objects such as human face, with no need of complete internal particulars, even lack of external boundary contour, people can still make out [6].

With progressive investigation on visual perception in psychological sense, the physiological process at the back of Gestalt principle is gradually revealed. With better understanding about and the appropriate use of Gestalt theory, artists are able to improve artistic expression speed and accuracy by foreseeing observers' psychological reaction when they're designing visual effect. In recent years, visual designers utilize related knowledge of visual perception like analyzing, arranging and organizing way in the brain in the design of visual effect, which is made to affect directly the perception of spectators so that they will react immediately after looking into information implications. Studies and theories on form psychology are closely associated with art visual design. Page 
layout, box, color block, internema etc. are common design instruments employed by designers to try to get viewers' particular feelings [7-8].

In the paper, we make use of those features mentioned to raise one algorithm for producing hiding images. It extracts edges of background images and hiding objects and their strength; then, match based on boundary strength as to find out in background images a proper location for objects to get embedded, scale and rotate there. The matching considers not only the position and also the strength of boundary feature points. On the basis of such information, one energy function is defined for form matching.

\section{Form Matching and Image Fusion}

Form matching methods in image processing are generally based on brightness or characteristics. The brightness-based methods regard the intensity of each image pixel as feature descriptor; however, intensity values may be influenced by other factors, e.g. object's posture or illumination change. Feature-based methods describe object shape with spatial domain and descriptor like SIFT referring to feature vectors of low dimension. Others use image contour relationship as shape descriptor, such as Hausdorff to represent the distance between two images. Shape context has translation and scaling invariability and that it's widely used for shape matching, especially in the field of alphabet recognition. Researchers introduced geometric fuzziness to realize template matching during the affine deformation. Over these years, shape matching methods based on characteristics are becoming popular. However, a majority of works are contributed to the complete similarity measurement between two complete shapes, very few about partial similarity measuring between them.

For the existing image fusion algorithms, the main objective is how to cause fewer defects when inserting one image to another one. A traditional method is Alpha fusion algorithm. But when the illumination of two images differs, obvious faults will occur. Perez [9] fused images by computing Poisson equation in one gradient domain, dealing well with transparency and partial object, and eliminating fusion defects as well; Jia [10]improved the method by optimizing fused boundaries; then Chen [11] improved the method further to obtain more natural fusion effect; Farbman [12] acquired the approximate fusion result without solving the complex Poisson equation.

Some works introduced texture synthesis method to achieve similar image fusion effect. Fang [13] applied the surface normal of restoring one image from another image to produce embossment effect; Ritter [14] presented a texture rendering system, which can merge texture strokes to have natural boundary effect; Oliva [15] stated one method to generate combined images by overlaying two images at different spatial scales; when a viewer watches the image at different distance, there will have two different recognitions of the image; Chu [16] developed a texture synthesis method to conceal objects in background images; the method can remove initial texture details of foreground objects and replace them with surrounding background textures.

\section{Generation of Hiding Images}

Hiding image is an artistic expression form. In the background image, one or more minor objects is hidden. When a viewer watches the image, the background can be directly discerned. However, after looking through features in background, a viewer can identify if other objects hide in the image. Those hiding objects make use of features in background image. Figure 1 shows two hiding images created by artists. 

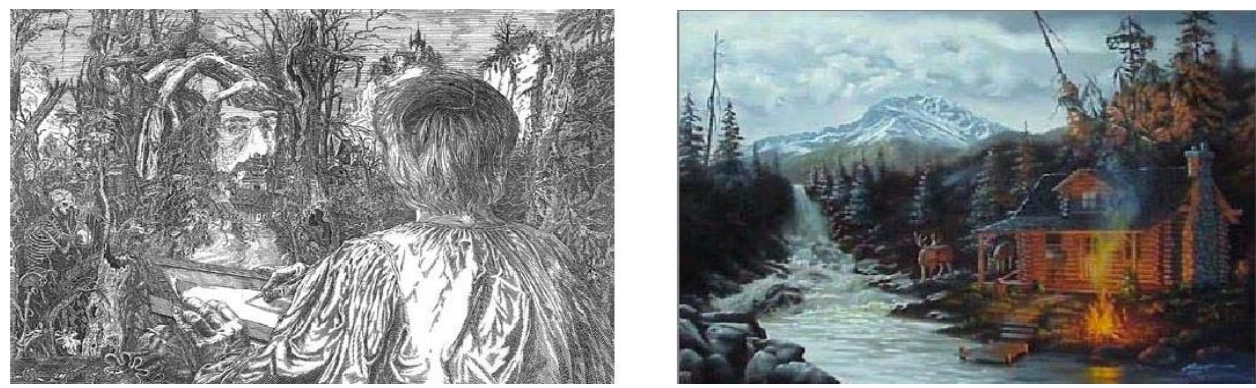

Figure 1. Hiding Images

\subsection{Object Edge Matching Based on Edge Strength}

For two images as input, it firstly needs to perform necessary pre-processing. Since human beings are more sensitive to the image brightness channel change than to the color distortion channel change. We fetch the luminance $\mathrm{Y}$ channel of two images in $\mathrm{YCrCb}$ color space for subsequent operation. Here we use $\mathrm{O}$ and $\mathrm{H}$ to respectively represent the luminance channel image of object and background images.

Traditional object matching methods are based on object's outer outline. We attempt to consider weak boundaries of an object in the matching procedure. Even final matching result is not very good in the outer shape, internal edges can provide acceptable matching result. If such a matching result is implanted to an object, the audience can replenish object's missing edges with its own priori knowledge so as to recognize objects. The goal of the paper is to find a transformed $\mathrm{T}$ of object $\mathrm{O}$ to minimize edge difference energy between deformed objects and background images. We use Sobel operator to fetch from $\mathrm{O}$ and $\mathrm{H}$ in the strength $[0,255]$.

Energy $E_{T}$ describes the edge difference between background image $\mathrm{H}$ and distorted object image $O_{T}$. To optimize the energy, it's necessary to determine the deformed T; use $e^{H}$ to represent the edge image fetched by $\mathrm{H}$ and $e_{T}^{O}$ to represent edge image fetched by $O_{T}$. In order to improve the position flexibility of edges in the matching, these edge diagrams are expansively performed by a simple $3 * 3$ rectangle operator i.e. $e^{H}$ and $e_{T}^{o}$ to arrive at respectively $E^{H}$ and $E_{T}^{o}$. It is shown in Figure 2.
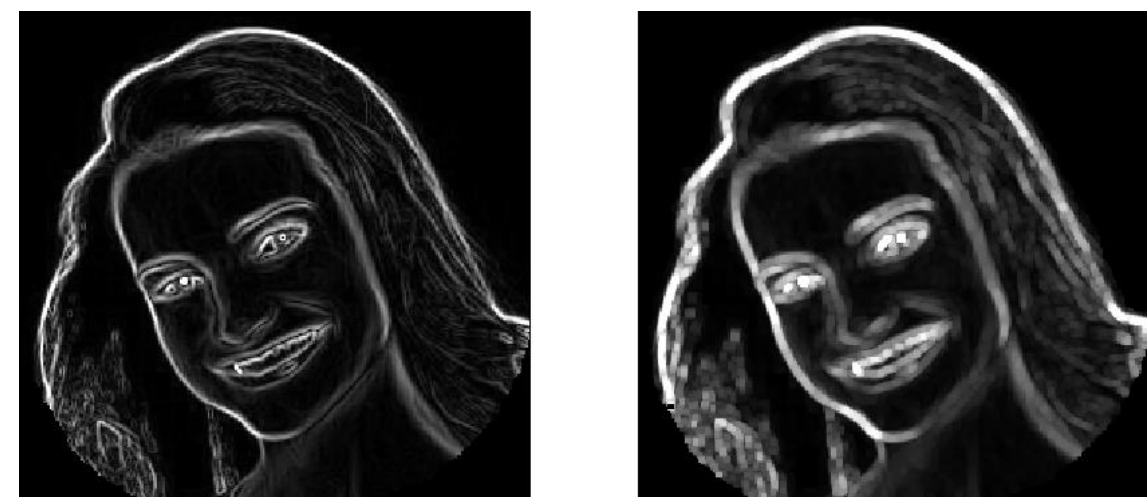

Figure 2. Edge Image of Object

The energy function $\mathrm{T}$ for describing image differences of expansive edges is defined as:

$$
\mathrm{E}_{T}=\frac{1}{S(\Omega)} \sum_{p \in \Omega} \mathrm{E}_{T}(p)
$$


Where, $S(\Omega)$ is defined as the mask area, and the matching energy per pixel $\mathrm{p}$ is defined as:

$$
\mathrm{E}_{T}(p)=\frac{\left|E^{H}(p)-E_{T}^{o}(q)\right|}{E^{H}(p)+E_{T}^{o}(q)} \frac{255}{\max _{p}\left(E^{H}(p), E_{T}^{o}(q)\right)} \sin (\gamma)
$$

We use a homogeneous transformation matrix $\mathrm{T}$ to support translation, rotation and scaling:

$$
T=\left(\begin{array}{ccc}
s \cos \theta & -s \sin \theta & \Delta x \\
s \sin \theta & s \cos \theta & \Delta y \\
0 & 0 & 1
\end{array}\right)
$$

However, searching completely the 4D parametric space is a very time-consuming task. We can confine it to a smaller range with the use of some strategies, as displayed by Algorithm 1. When for layer-by-layer transmission, several backup locations are passed to avoid falling into the local optimum. Figure 3 gives a matching instance. In Figure 3(c), energy differentiation is compared between background image and deformed image under the best matching circumstances.

Algorithm1 Multi scale shape matching

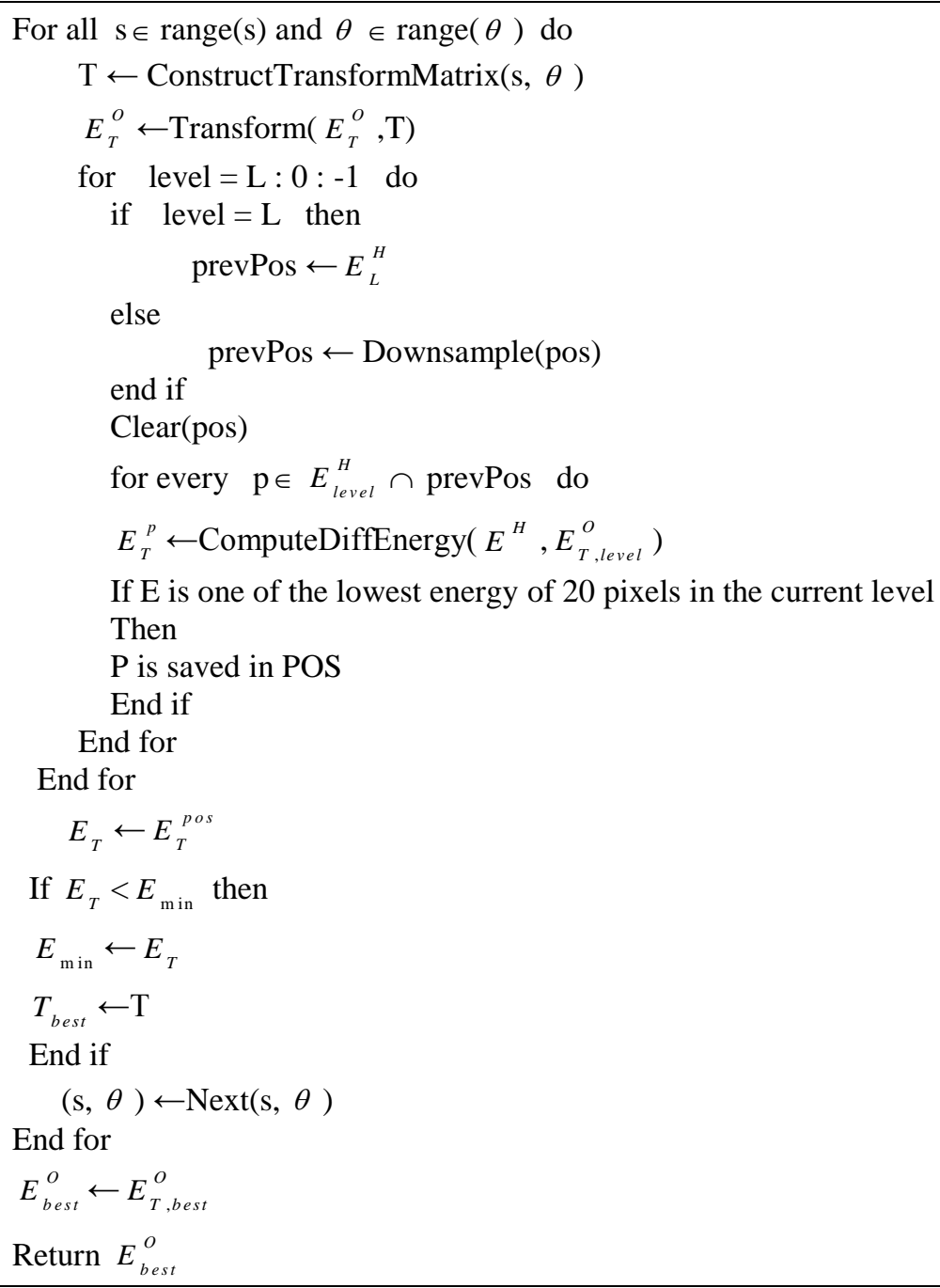

If $\mathrm{E}$ is one of the lowest energy of 20 pixels in the current level

Then

$\mathrm{P}$ is saved in POS

End if

End for

End for

$$
E_{T} \leftarrow E_{T}^{p o s}
$$

If $E_{T}<E_{\text {min }}$ then

$E_{\text {min }} \leftarrow E_{T}$

$T_{\text {best }} \leftarrow \mathrm{T}$

End if

$(\mathrm{s}, \theta) \leftarrow \operatorname{Next}(\mathrm{s}, \theta)$

End for

$E_{\text {best }}^{o} \leftarrow E_{T, \text { bes }}^{o}$

Return $E_{\text {best }}^{o}$ 

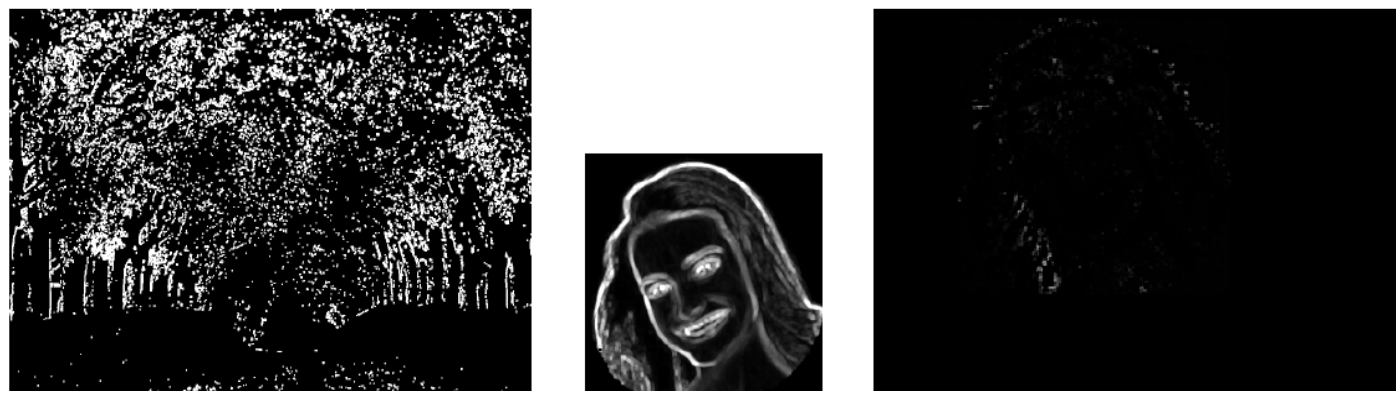

(a) Edge Expansion Image (b) Edge Expansion Image of Deformable Objects (c) Matching Energy

\section{Figure 3. The Edge Image is Optimized to Get Combination Edge Image}

\subsection{Fusion Algorithm for the Object Embedding Background Images}

When discovering the best position and direction of an object hiding in background image, we use Poisson fusion technology to generate the ultimate hiding image. For traditional Poisson fusion method, we need to solve Equation (4) as to determine each pixel luminance value $L(p)$ of $O_{T}$ in the masking $\Omega$ as follows:

$$
\left|N_{p}\right| L(p)-\sum_{q \in N_{p} \cap \Omega} L(q)=\sum_{q \in N_{p} \cap \partial \Omega} H(q)+\sum_{q \in N_{p}} v_{p q}
$$

But, traditional Poisson fusion algorithm does not work well for problems here. As $v_{p q}$ is used to control gradient values in the masking, images after fusion have identical gradients to object images. If the background image has abundant texture details but object image lack of them, the traditional Poisson fusion method will produce worse results. Besides, our shape matching algorithm depends on edge strength to find out the optimal matching position of the background and object images; while object insertion procedure requires ignoring strong edges with bad matching and enhancing edges with better matching, so that those edges will stand out from the background image after fusion.

To solve problems here, we improved Poisson fusion method as for object insertion. We take into account the gradient of background image $\mathrm{H}$, object image $O_{T}$ and object edge image $E_{T}$ to decide the $v_{p q}^{\prime}$ item in the Poisson equation, like the following:

$$
\begin{aligned}
v_{p q}^{\prime}= & (1-\alpha)(H(p)-H(q))+\alpha \beta\left(O_{T}(p)-O_{T}(q)\right) \\
& +\alpha(1-\beta)\left(E_{T}(p)-E_{T}(q)\right)
\end{aligned}
$$

\section{Experiment Design and Discussion}

Even for a skilled artist, it takes a long time to create an interesting hiding picture. When creating such a graph, the artist needs to draw out firstly outline sketches of background image and for-insertion object; and then attempts to compose them into one entity. When adding graphic details, the artist must be very careful to try to strike balance between hiding and embedded objects. The proposed algorithm provides one automatic method to realize that purpose. Figure 4-5 list some examples which are generated by this technique. 


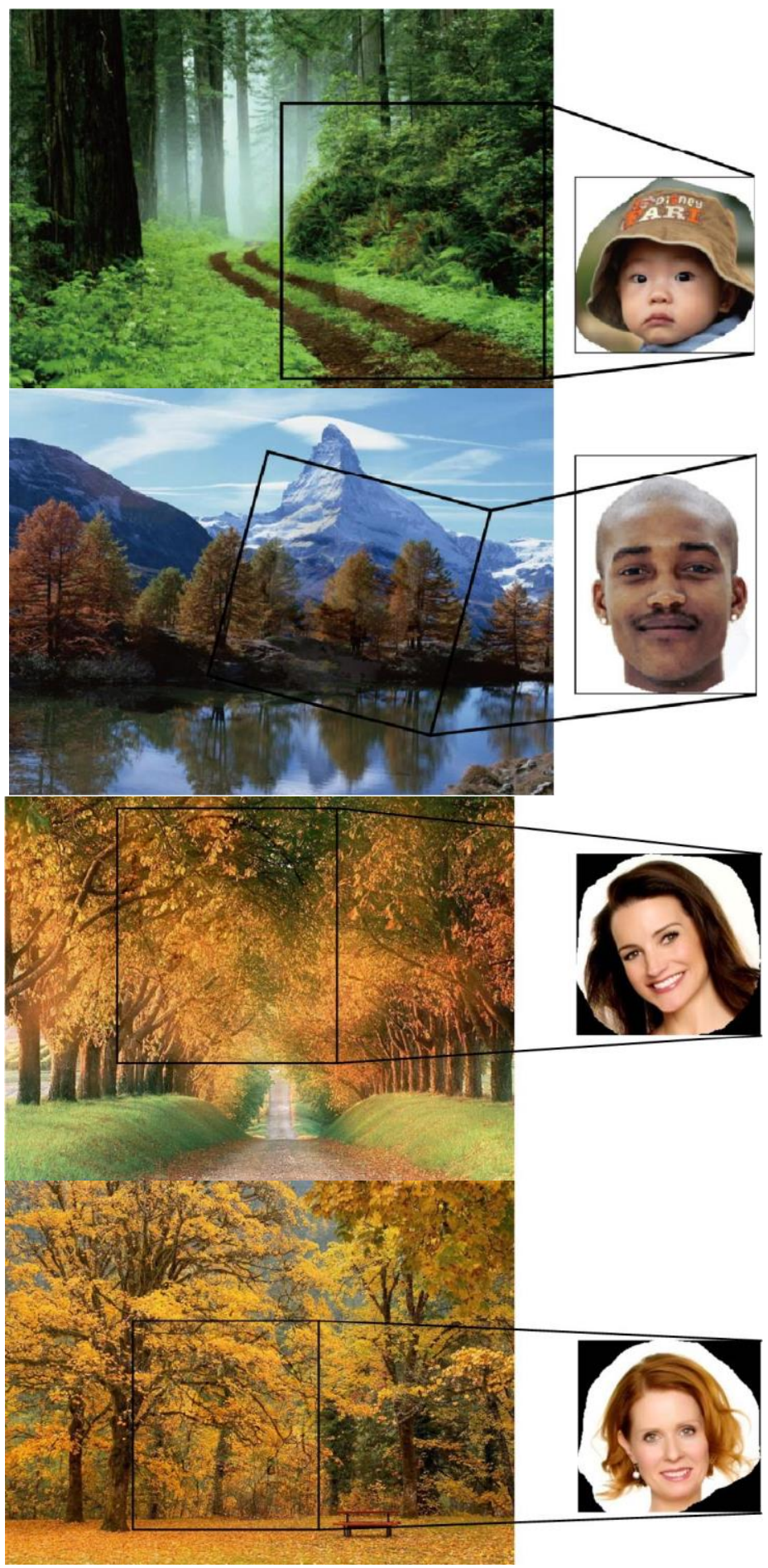

Left: Algorithm 1 Automatically Generated Hidden Images. Right: Embedded Objects

Figure 4. Some Hidden Image Results 

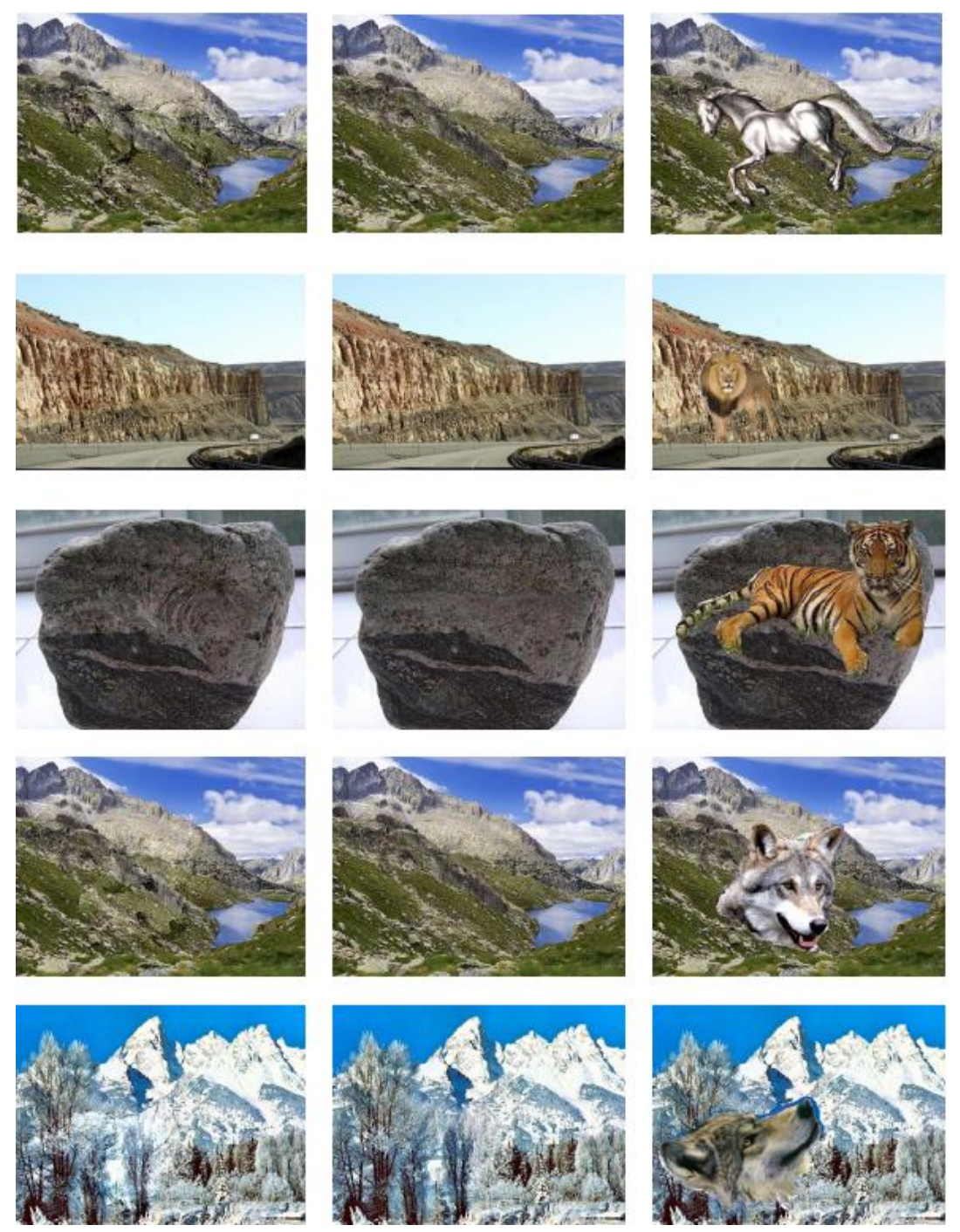

Left: Algorithm 1 Automatically Generated Hidden Images. Right: Embedded Objects.

Figure 5. Some Hidden Image Results

All examples cited in the paper are produced by one PC with Intel Core $22.10 \mathrm{GHz}$ and $2 \mathrm{~GB}$ internal memory. Table1 shows parameter $\theta$ and $\mathrm{s}$, including their step length and computing time required for generating the final image. It's observed that shape matching consumes most time. Object embedding requires shorter time because it's based on the Poisson fusion method. Shape matching speed is slow because we need to traverse lots of positions where the object image may be placed. By pre-assigning some areas by users, the time will effectively be reduced.

Figure 6 shows different generative results with different parameter $\alpha$ and $\beta$ for the background and object images in the same group. With diminution of $\alpha$, the embedded object becomes more imperceptible; while with growing $\beta$, the embedded object is manifested by its edges. In graph 6 , when $\alpha<0.4$, the embedded object almost disappears totally; when $\alpha>0.8$, it becomes too discernible. 
Table 1. Hidden Image Algorithm Performance

\begin{tabular}{|l|l|l|l|l|l|l|l|}
\hline Image & $\begin{array}{l}\text { Background } \\
\text { map }\end{array}$ & Object & $\theta$ & $\mathrm{s}$ & $\begin{array}{l}\text { Matching } \\
\text { time }\end{array}$ & $\begin{array}{l}\text { Embedding } \\
\text { time }\end{array}$ & $\begin{array}{l}\text { Total } \\
\text { time }\end{array}$ \\
\hline $\begin{array}{l}\text { Figure4 } \\
\text { (a) }\end{array}$ & $1024 \times 768$ & $\begin{array}{l}273 \times \mathrm{x} \\
272\end{array}$ & $-30^{\circ}: 30^{\circ}: 15^{\circ}$ & $1.0: 3.0: 0.2$ & $27 \mathrm{~s}$ & $5 \mathrm{~s}$ & $32 \mathrm{~s}$ \\
\hline $\begin{array}{l}\text { Figure4 } \\
\text { (b) }\end{array}$ & $700 \times 525$ & $\begin{array}{l}189 \times \\
249\end{array}$ & $-30^{\circ}: 30^{\circ}: 15^{\circ}$ & $0.6: 2.0: 0.2$ & $11 \mathrm{~s}$ & $1 \mathrm{~s}$ & $12 \mathrm{~s}$ \\
\hline $\begin{array}{l}\text { Figure4 } \\
\text { (c) }\end{array}$ & $1014 \times 718$ & $\begin{array}{l}263 \times \\
256\end{array}$ & $-30^{\circ}: 30^{\circ}: 15^{\circ}$ & $1.0: 3.0: 0.2$ & $26 \mathrm{~s}$ & $5 \mathrm{~s}$ & $31 \mathrm{~s}$ \\
\hline $\begin{array}{l}\text { Figure4 } \\
\text { (d) }\end{array}$ & $1280 \times 960$ & $\begin{array}{l}261 \times \mathrm{x} \\
244\end{array}$ & $-30^{\circ}: 30^{\circ}: 15^{\circ}$ & $1.0: 3.0: 0.2$ & $29 \mathrm{~s}$ & $3 \mathrm{~s}$ & $32 \mathrm{~s}$ \\
\hline
\end{tabular}
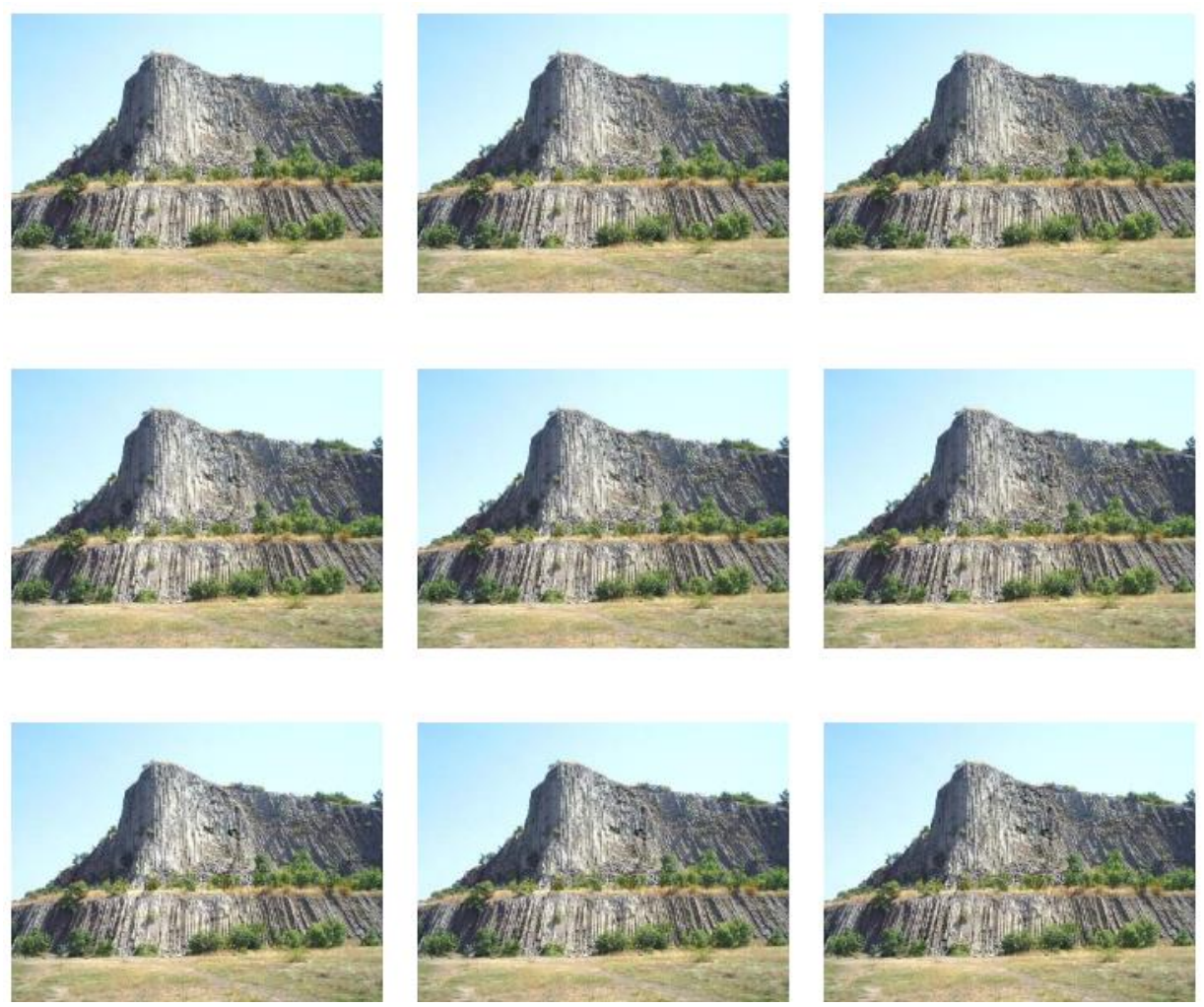

From Top to Bottom, $\alpha$ Fetchs 0.4, 0.6, 0.8; from the Left to the Right, $\beta$ Fetchs 0.4, 0.6, 0.8 .

\section{Figure 6. Hidden Images Using Different Embedding Parameters}

We compared results with Chu's algorithm [16]. Although Chu tried to embed objects in the background image, our solution differs greatly from his. In Chu's method, it removed texture details in the object image and composed new physical textures according to background image textures and object structure to ultimately generate camouflage images. When seeing those camouflage images, audiences have to put together many adjacent color blocks to identify hiding objects. In our method, it generates hiding images by overlaying and abandoning object edges. When audiences are watching them, based on detected edges, they recognize embedded objects consciously by making up missing edges and object outer profile. Figure 7 compares results by the proposed object embedding algorithm and Chu's texture synthesis method. What should be noted that in the method here, object's embedding position in the background image is chosen manually. For more sufficient comparison, we use the proposed method to generate two hiding images: one is got by the embedding algorithm in the same position, i.e. without 
searching the embedding position; the other is produced by the automatic shape matching method here.
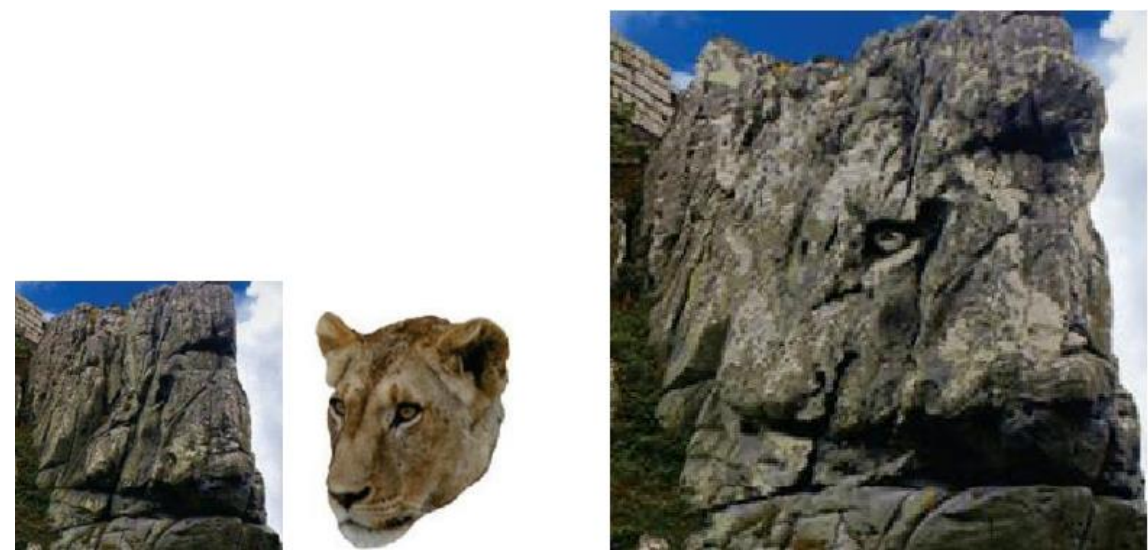

(a) Input Background and Embedded Object (b) Embedding Results of [16] Method
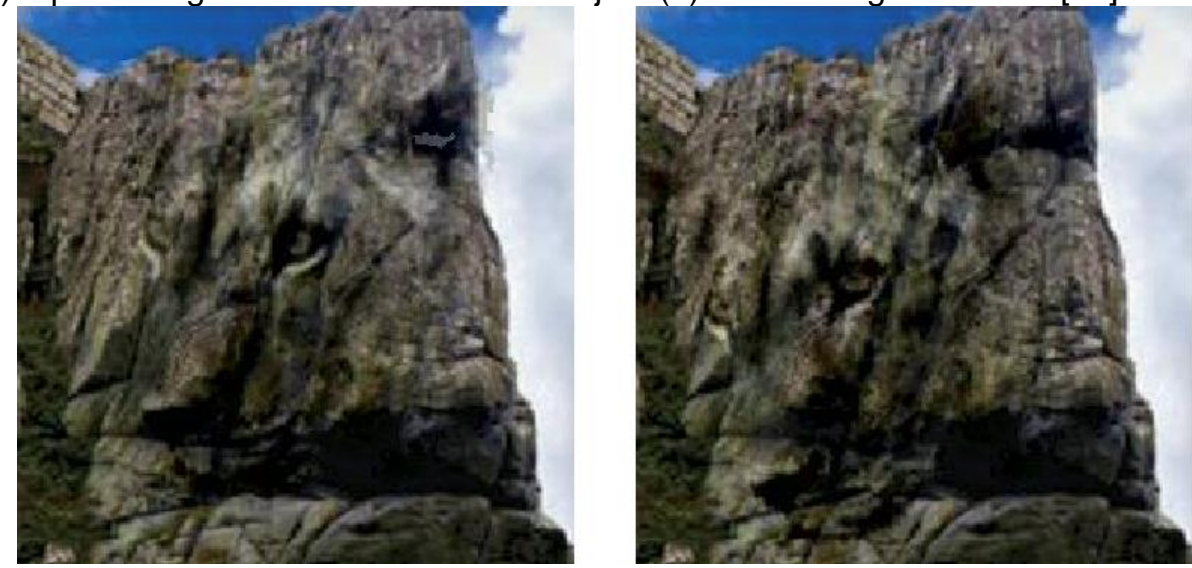

(c) In the Same Position as [16] to Adopt this Method to Embed Objects; (d) Find the Location and Embed the Object Automatically by this Method

\section{Figure 7. Comparison Between the Present Method and the [16] Method}

\section{Conclusion}

An automatic algorithm for generating hiding images is presented in the paper. By using shape matching and object embedding technologies, the algorithm considers edges and thus to reach a better effect by inserting objects into the background image. When an audience is watching background image, he can recognize embedded object. The method can perform well for object images with either rich or fewer textures.

\section{Acknowledgment}

This work was supported by the National Natural Science Foundation of China (Grant No.61303146).

\section{References}

[1] L. Lingyu, "Research on adaptive and rendering of human face images", South China University of Technology, (2014).

[2] H. Xi, "Study on the depth information of stereo image generation algorithm based on", Beijing University of Posts and Telecommunications, (2011). 
[3] N. Shengxiao, W. Sheng, Y. Jingjing and C. Gengsheng, "Completely based on edge information fast image segmentation algorithm", Journal of computer aided design and computer graphics, vol. 11, (2012), pp. 1410-1419.

[4] L. Xiuping, O. Xiantang and L. Zhilong, "A kind of edge and region information based level set image segmentation method", Journal of Dalian University of technology, vol. 5, (2008), pp. 754-758.

[5] L. Xuehui, W. Shandong, C. Yanyun and W. Enhua, "Drawing on the abstract lines based on feature flow", Chinese Journal of computer, vol. 3, (2014), pp. 611-620.

[6] Z. Fan, L. Xiaodong and Z. Wenjun, "Automatic generation method based on real scene of ink style image”, TV technology, vol. 15, (2014), pp. 249-252.

[7] K. Fanshu, A. Dong, J. Chao, Y. Fang, Z. Qi and W. Beibei, "Image interpolation algorithm based on edge information", Computer engineering and design, vol. 13, (2009), pp. 3157-3159.

[8] H. Ning and Z. Peng, "The method of dividing the image segmentation method based on the combination of edge and region information", Electronic Journal, vol. 10, (2009), pp. 2215-2219.

[9] P. Perez, M. Gangnet and A. Blake, "Poisson image editing", ACM Transactions on Graphics (Proceeding SIGGRAPH), vol. 22, no. 3, (2003), pp. 313-318.

[10] J. Jia, J. Sun and C. K. Tang, "Drag-and-Drop Pasting", ACM Transactions on Graphics, vol. 25, no. 3, (2006), pp. 631-637.

[11] T. Chen, M .M. Cheng and P. Tan, "Sketch2Photo: Internet Image Montage", ACM Transactions on Graphics (Proceeding SIGGRAPH), vol. 28, no. 5, (2009), pp. 124-133.

[12] Z. Farbman, G. Hoffer and Y. Lipman, "Coordinates for Instant Image Cloning", ACM Transactions on Graphics, vol. 28, no. 3, (2009), pp. 67-74.

[13] H. Fang and J. Hart, "Textureshop: texture synthesis as a photograph editing tool”, ACM Transactions on Graphics (Proceeding SIGGRAPH), vol. 23, no. 3, (2004), pp. 354-359.

[14] L. Ritter, W. Li and B. Curless, "Painting with texture. Eurographics Symposium on Rendering", (2006).

[15] A. Oliva, A. B. Torralba and P. G. Schyns, "Hybrid images", ACM Transactions on Graphics, vol. 25, (2006), pp. 527-532.

[16] H. K. Chu, W. H. Hsu and N. J. Mitra, "Camouflage Images", ACM Transactions on Graphics, vol. 29, no. 3 , (2010), pp. 51-60.

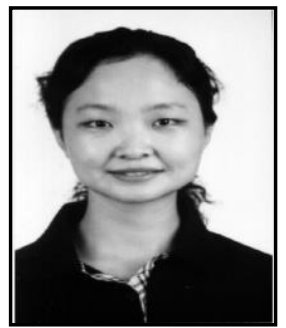

\section{Authors}

Yanqiu Liu, she received her B.S degree from Zhengzhou University and received her M.S degree from Jiangnan University. She is a lecturer in China Jiliang University. Her research interests include Computer graphics image processing, Machine vision, Multimedia technology.

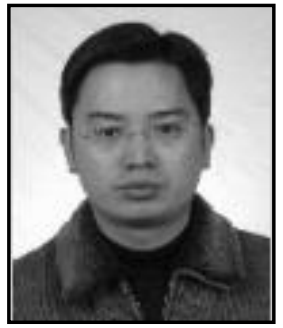

Xiuhui Wang, he received his B.S degree from Zhengzhou University. He received his M.S degree and Ph.D. degree all from Zhejiang University. He is an associate professor in China Jiliang University. His research interests include Computer vision, Graphics image processing, Virtual reality technology. 